\title{
Penile rehabilitation should not be the norm for patients post-radical prostatectomy
}

\author{
Jay Lee, MD
}

$\mathrm{D}$ espite advances in surgical techniques, erectile dysfunction (ED) still remains a significant problem for men after radical prostatectomy (RP). Recovery of sexual function impacts quality of life and satisfaction with treatment. ${ }^{1}$ The etiology of ED after surgery is thought to be secondary to neurapraxia of the cavernous nerves, leading to decreased nitric oxide production and reduced arterial dilation. ${ }^{2}$ Some patients will recover some or all of their presurgery erectile function, but this recovery is a slow process that can take up to 4 years, and others will never fully recover to their presurgery levels. ${ }^{3}$ It is only natural that patients would like to recover more quickly and more completely.

Pilot studies have been performed in the past to see if "penile rehabilitation" would aid in the earlier return of erections sufficient for sexual activity post-RP. The landmark paper by Montorsi and colleagues ${ }^{4}$ compared regular administration of alprostadil intracavernosal injections (ICls) versus no injection therapy. Six months post-RP, spontaneous sexual erections were reported in significantly more men who had undergone routine $\mathrm{ICl}$ compared with those who did not $(67 \%$ v. $20 \%$; $p<0.01)$. Several animal studies examined the use of phosphodiesterase-5 (PDE5) inhibitors for prevention of fibrosis and loss of smooth muscle after cavernosal nerve injury. ${ }^{5,6}$ Although results in these animal studies seem promising, they have not been conclusively demonstrated in humans. One study, conducted by Padma-Nathan and colleagues, ${ }^{7}$ investigated the use of nightly sildenafil versus placebo for 36 weeks in a small group of men post$\operatorname{RP}(n=76)$. The results showed that men who received nightly sildenafil (50 or $100 \mathrm{mg}$ ) after surgery had an increased return of normal spontaneous erectile function $(27 \% \mathrm{v} .4 \%$ in the placebo group).

It is clear that there are significant issues with the aforementioned clinical studies. The most obvious one is the small sample sizes, which has a profound impact on the robustness of the data. As well, the studies were performed in 1 or a few centres of excellence, thereby reducing the applicability to community urologists and general patients undergoing RP. The studies included only men with normal erectile function preoperatively, so very little is known whether penile rehabilitation has any efficacy in men with pre-existing ED. These studies also only allowed men with "successful" bilateral nerve-sparing RP; we have no evidence of the benefit of penile rehabilitation for patients who underwent unilateral nervesparing or non-nerve-sparing RP. Nevertheless, many urologists adopted penile rehabilitation for the post-RP population. In terms of the rehabilitation, there was no consensus as to the best medications (oral v. injection), dosing (quarter v. half v. full dose) or timing of the start of treatment.

Teloken and colleagues ${ }^{8}$ examined the practice patterns for penile rehabilitation in 41 countries. Of 307 physicians surveyed, $83.7 \%$ stated that they recommended some form of penile rehabilitation. However, the therapeutic agents used varied from daily PDE5 inhibitors (varying doses and compounds), ICls, vacuum-assisted erectile devices (VEDs) or combinations. For those who did not employ penile rehabilitation, cost and lack of evidence were cited as the main reasons. Thus many questions still remain regarding the timing, dosing, efficacy and even the validity of penile rehabilitation.

Recently, Montorsi and coauthors ${ }^{9}$ published the results of the REINVENT (Recovery of Erections: INtervention with Vardenafil Early Nightly Therapy) study. This was a multicentre, multinational, double-blind, placebo-controlled, randomized study of post-RP patients comparing nightly vardenafil $(10 \mathrm{mg})$ versus on-demand vardenafil (10-20 mg) versus placebo for 36 weeks. The study had the largest sample size of any published penile rehabilitation study to date $(n=423)$ and provides the strengths in design (large population, multi- 
centre, blinded, inclusion of an on-demand arm) that the previous studies lacked. Men with normal preoperative erectile function undergoing bilateral nerve-sparing RP were included. Validated questionnaires such as the International Index of Erectile Function (IIEF) as well as sexual diary reporting (Sexual Encounter Profile [SEP] 2, SEP3) were used to assess sexual function. During the initial 36week double-blind phase, those men who were randomized to on-demand therapy had significantly better IIEF scores as well as significantly greater ability to complete sexual intercourse when compared with men randomized to nightly dosing or placebo (Fig. 1). Patients were also evaluated at about 11 months post-RP. During this 2-month single-blind phase, all patients regardless of previous randomization were given placebo to assess erectile function without the aid of PDE5 inhibitors. If a true rehabilitation effect was established, then it was assumed that men previously randomized to the nightly dosing arm would have significantly better erections than those randomized to placebo or on-demand dosing. However, this was not the finding (Fig. 1, Fig. 2). Regardless of whether men had 36 weeks of placebo, nightly dosing, or on-demand dosing, there was no difference in spontaneous sexual erections. Thus no rehabilitation effect was seen.

Even with what some may consider a negative result, positives are found for post-RP patients. In the REINVENT study, significantly more men who were randomized to on-demand vardenafil during the 36 weeks were able to obtain sexual erections sufficient to complete intercourse compared with the other groups. As well, for those men who did not take early therapy (whether nightly or on-demand in the first 36 weeks), they still did as well when taking on-demand vardenafil 1 year later. Thus for patients who are not ready to resume sexual activity (e.g., owing to incontinence), they are not condemned to worsening sexual function in the future. The REINVENT study is by far the best-designed study published to date regarding penile rehabilitation in post-RP ED.

What does this mean for our patients? The early studies definitely suggested some plausible treatment options for ED post-RP. However, patients incur significant financial expenditure without clear, clinical benefit. There is also a risk of disappointing patient expectations. In addition, there is always the question of whether patients will be compliant with taking an ED medication daily,

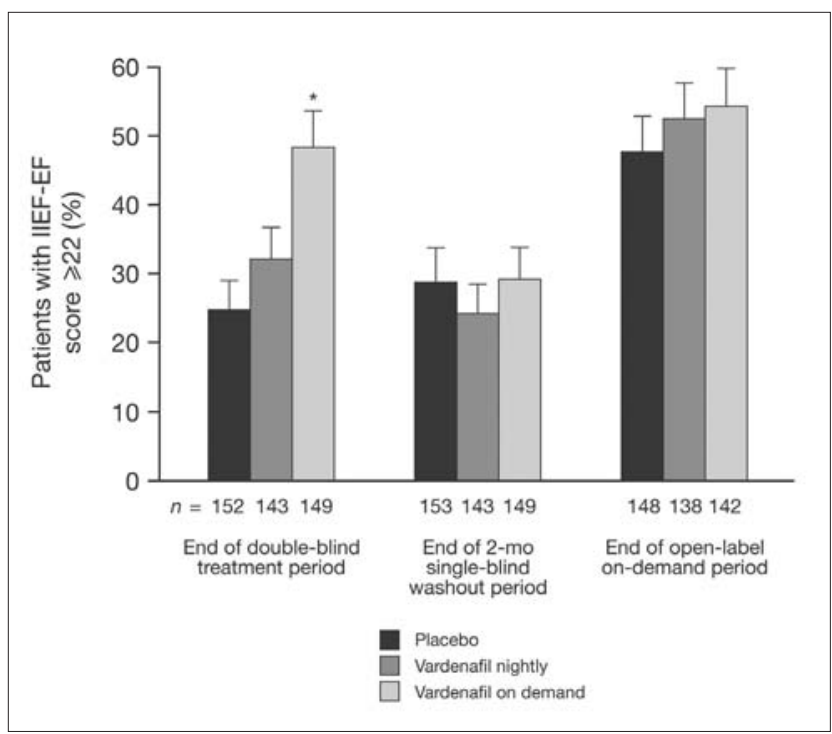

Fig. 1. Results of the REINVENT study, double-blind phase. Men who achieved International Index of Erectile Function, erectile function (IIEFEF) scores of greater than $22 .{ }^{*} p<0.001$ for comparison of vardenafil on-demand versus placebo.

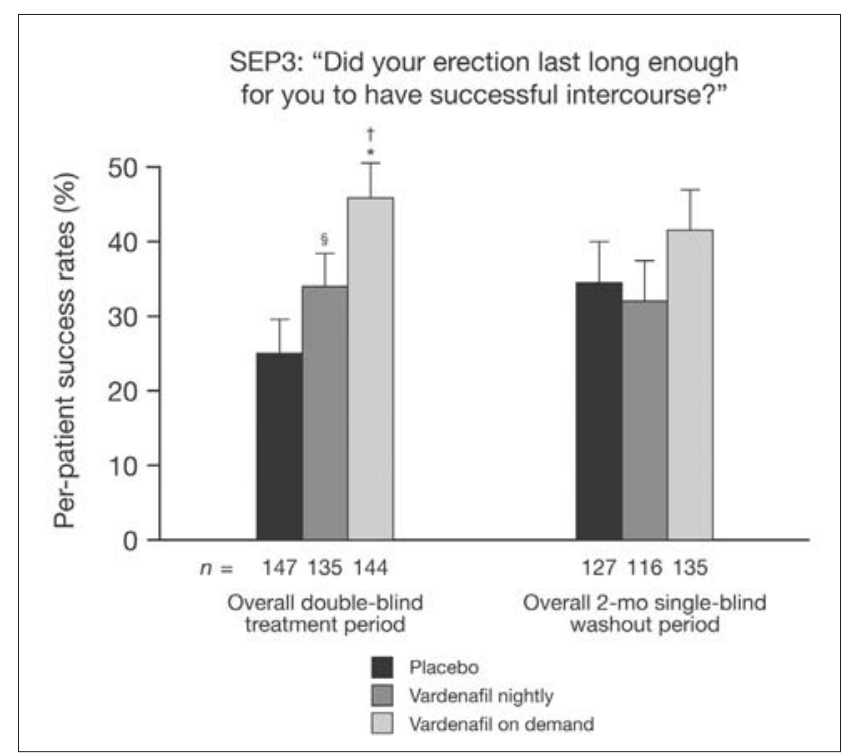

Fig. 2. Results of the REINVENT study, successful intercourse, doubleblind phase and single-blind phase. ${ }^{9}$ SEP3 = Sexual Encounter Profile, question $3 .{ }^{*} p<0.001$ for the comparison of vardenafil on-demand versus placebo. $\S p=0.034$ for the comparison of vardenafil nightly versus placebo. $\dagger p=0.011$ for the comparison of vardenafil on-demand versus vardenafil nightly. 
as noncompliance with other medications, such as antihypertensives, is well known. We all want to provide for our patients the best care established on evidence-based medicine. However, there is not sufficient evidence currently to recommend daily PDE5 inhibitors as the standard of care for our post-RP patients suffering from ED. Fortunately, there are still safe and effective treatment options, including PDE5 inhibitors, ICls, intraurethral therapy, VEDs and penile implants. Using these clinically proven on-demand treatment options, patients will be able to resume sexual activity when they are ready, which will provide patients with satisfaction and decrease their frustration. Some of these treatments have been demonstrated to be effective for men regardless of preoperative erectile function, whereas penile rehabilitation has only been investigated in men with normal preoperative erectile function. Until we have further evidence, daily PDE5 inhibitors should not be the norm for all patients with ED post-RP.

Clinical Lecturer, University of Calgary, Calgary, Alta., Director of the Sexual Health Clinic, The Prostate Cancer Institute, Calgary, Alta.

The positions provided in the Point/Counterpoint series are presented as general information and do not necessarily reflect the personal opinions of the authors.

This article has been peer reviewed.
Competing interests: None declared.

\section{References}

1. Litwin MS, Hays RD, Fink A, et al. Quality-of-life outcomes in men treated for localized prostate cancer. JAMA 1995;273:129-35.

2. Burnett AL. Rationale for cavernous nerve restorative therapy to preserve erectile function after radical prostatectomy. Urology 2003;61:497-7.

3. Rabbani F, Stapleton AMF, Kattan MW, et al. Factors predicting recovery of erections after radical prostatectomy. J Urol 2000;164:1929-34.

4. Montorsi F, Guazzoni G, Strambi LF, et al. Recovery of spontaneous erectile function after nerve-sparing radical retropubic prostatectomy with and without early intracavernous injections of alprostadil: results of a prospective, randomized trial. J Urol 1997;158:1408-10.

5. Ferrini MG, Davila HH, Kovanecz I, et al. Vardenafil prevents fibrosis and loss of corporal smooth muscle that occurs after bilateral cavernosal nerve resection in the rat. Urology 2006;68:429-35.

6. Kovanecz I, Rambhatla A, Ferrini M., et al. Long-term continuous sildenafil treatment ameliorates corporal veno-occlusive dysfunction (CVOD) induced by cavernosal nerve resection in rats. Int I Impot Res 2008;20:202-12.

7. Padma-Nathan H, McCullough AR, Levine LA, et al. Randomized, double-blind, placebo-controlled study of postoperative nightly sildenafil citrate for the prevention of erectile dysfunction after bilateral nerve-sparing radical prostatectomy. Int I Impot Res 2008;20:479-86.

8. Teloken PE, de Mesquita GG, Montorsi F, et al. Post-radical prostatectomy pharmacological penile rehabilitation: current practice status. J Sex Med 2006;3 (suppl 5):394.

9. Montorsi F, Brock G, Lee J, et al. Effect of nightly versus on-demand vardenafil on recovery of erectile function in men following bilateral nerve-sparing radical prostatectomy. Eur Urol 2008;54:924-31.

Correspondence: Dr. Jay Lee, 404-1011 Glenmore Trail SW, Calgary AB T2V 4R6; jayclee77@hotmail.com

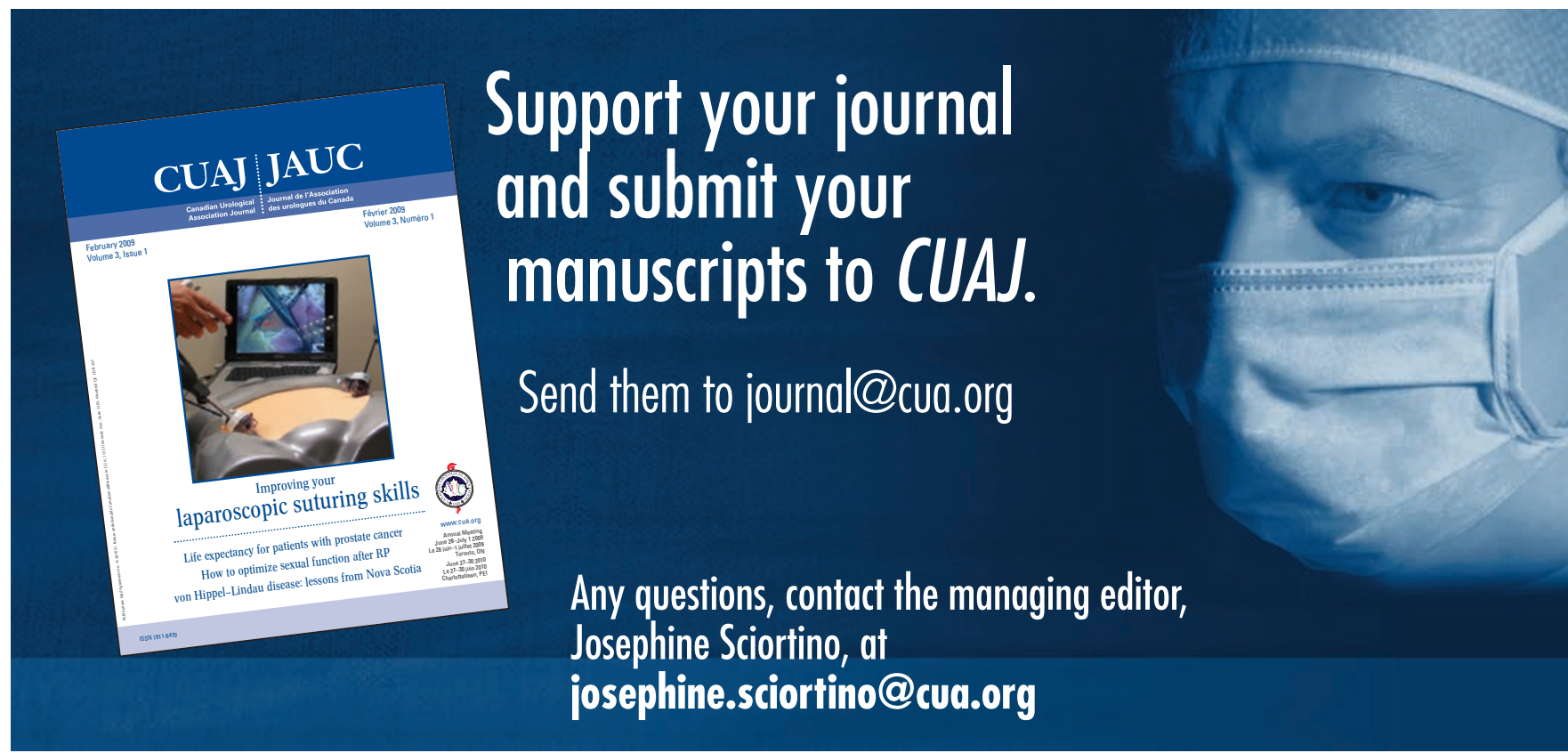

